\title{
Co-culture of bone marrow stem cells and macrophages indicates intermediate mechanism between local inflammation and innate immune system in diabetic periodontitis
}

\author{
JIA WANG ${ }^{1}, \mathrm{HAO} \mathrm{LI}^{2}, \mathrm{BO} \mathrm{LI}^{2}$, QIULIN GONG${ }^{2}, \mathrm{XINMIN} \mathrm{CHEN}^{2}$ and $\mathrm{QI} \mathrm{WANG}^{2}$ \\ ${ }^{1}$ Department of Stomatology, Chengdu Fifth People's Hospital, Chengdu, Sichuan 611130; \\ ${ }^{2}$ State Key Laboratory of Oral Diseases, West China Hospital of Stomatology, \\ Sichuan University, Chengdu, Sichuan 610041, P.R. China
}

Received November 5, 2014; Accepted December 18, 2015

DOI: $10.3892 / \mathrm{etm} .2016 .3386$

\begin{abstract}
Diabetic periodontitis (DP), which has been shown to cause alveolar bone loss, is among the most common complications associated with diabetes. The precise mechanisms underlying alveolar bone loss in patients with DP remain unclear. Therefore, the present study established a co-culture system of bone marrow stem cells (BMSCs) and macrophages, in order to investigate the potential mechanisms underlying DP-associated alveolar bone loss in vitro. In addition, Porphyromonas gingivalis (PG) periodontal infection and high glucose levels were used to induce DP in mice. The present study evaluated the protein expression levels of various chemokines and the migration of BMSCs and macrophages. The protein expression levels of extracellular signal-regulated kinase 1 and 2, c-Jun N-terminal kinase and p38 mitogen-activated protein kinase (MAPK) were significantly increased in the BMSCs exposed to high glucose and PG, which may have been due to the activation of MAPK. In addition, DP induction in mice was associated with the release of chemokine (C-C motif) ligand 2 (CCL2) from BMSCs and the secretion of chemokine (C-C Motif) receptor 2 (CCR2) and tumor necrosis factor- $\alpha$ from macrophages, which was associated in turn with enhanced adhesion and chemotaxis of macrophages. The results of the present study suggested that DP led to the upregulation of CCL2 in the periodontal tissues and enhanced macrophage infiltration via the CCL2/CCR2 axis, which in turn promoted alveolar bone loss.
\end{abstract}

Correspondence to: Dr Xinmin Chen, State Key Laboratory of Oral Diseases, West China Hospital of Stomatology, Sichuan University, $143^{\text {rd }}$ Section S Renmin Road, Chengdu, Sichuan 610041, P.R. China

E-mail: xinminchen@yeah.net

Key words: diabetic periodontitis, chemokine (C-C motif) ligand 2, tumor necrosis factor- $\alpha$, alveolar bone loss

\section{Introduction}

Periodontal disease (PD) is an infection-driven chronic inflammatory disease characterized by the resorption of the tooth-supporting alveolar bone (1). PD is typically induced by bacteria that colonize the tooth surface and gingival sulcus; however, the host immune response is hypothesized to serve a crucial function in the breakdown of connective tissue and bone, and is therefore considered to be a key feature of the disease process (2). In addition, an intermediate mechanism involving cytokines, including chemokines and innate or acquired immune cytokines, has been hypothesized to link the bacterial stimulation of the immune system with tissue destruction (3). Previous studies have reported that macrophage-derived chemokines were elevated in the destructive and reparative phases of periodontitis, and that the chemokines were crucially involved in the recruitment of macrophages (4). In addition, the cytokines may have directly or indirectly regulated the alveolar bone loss process (5).

In a previous study, the $\mathrm{C}-\mathrm{C}$ group chemokine monocyte chemoattractant protein-1 (MCP-1), which is also known as chemokine (C-C motif) ligand (CCL) 2, demonstrated chemotactic effects on lymphocytes and monocytes; suggesting that MCP-1 is a major signal for triggering the chemotaxis of mononuclear leukocytes (6). In addition, it has previously been demonstrated that the activation of the nuclear factor- $\kappa \mathrm{B}$, mitogen-activated protein kinase (MAPK) and Akt pathways in bone marrow stem cells (BMSCs), by high glucose levels or inflammation, induces the expression of interleukin (IL)-1 $\beta$, IL-6, CCL2, CCL5, chemokine (C-X-C motif) ligand (CXCL) 1 and CXCL5 via WNT5A (7).

The spatial organization of tissues and cell-cell interactions have been disregarded in the majority of available in vitro models. However, co-culturing techniques have recently been developed in order to enhance the similarities between cell cultures and in vivo systems $(8,9)$. Co-culture permits the free exchange of signaling molecules, including proteins, carbohydrates and other small molecules (10-12). Therefore, co-culture may be considered an alternative approach for mimicking the BMSC microenvironment in tissue culture. In addition, co-culture may permit the determination of the intermediate 
mechanism between local inflammation caused by bacterial infection and host immune responses in diabetic periodontitis (DP). The present study aimed to investigate the mechanisms underlying alveolar bone loss in patients with DP, using a co-culture system consisting of BMSCs and macrophages, and a mouse model of DP.

\section{Materials and methods}

Reagents. Tissue culture materials were obtained from Corning Incorporated (Corning, NY, USA). The chemicals and reagents used in the present study and their suppliers were as follows: Streptozotocin (STZ) was purchased from Sigma-Aldrich (St. Louis, MO, USA); the enzyme-linked immunosorbent assay (ELISA) kit for tumor necrosis factor (TNF)- $\alpha$ was obtained from Cusabio Biotech Co., Ltd. (Wuhan, China); the ReadyPrep Protein Extraction kit, nitrocellulose membranes and SuperSignal West Pico Chemiluminescent Substrate were obtained from Bio-Rad Laboratories, Inc. (Hercules, CA, USA). The antibodies used in the present study were as follows: Rabbit anti-glyceraldehyde-3-phosphate dehydrogenase (GAPDH) polyclonal antibody (1:300; sc-25778), mouse anti-c-Jun N-terminal kinase (JNK) monoclonal antibody (mAb; 1:200; sc-7345), mouse anti-phosphorylated (p)-JNK mAb (1:200; sc-6254), mouse anti-extracellular signal-regulated kinase (ERK) mAb (1:200; sc-376852), mouse anti-p-ERK mAb (1:200; sc-377400), mouse anti-p38 mAb (1:200; sc-81621), mouse anti-p-p38 mAb (1:200; sc-7973), mouse anti-CCL2 mAb (1:200; sc-1785), mouse anti-CCR2 mAb (1:200; sc-46862), and goat anti-rabbit (1:3,000; sc-2030) and anti-mouse (1:2,000; sc-2005) horseradish peroxidase-conjugated secondary antibodies (Santa Cruz Biotechnology, Inc., Dallas, TX, USA).

Animals and grouping. A total of 30 specific-pathogen-free grade 4-week-old C57BL/6 wild-type female mice, weighing $\sim 22 \mathrm{~g}$, were obtained from the Experimental Animal Laboratory of Sichuan University (Chengdu, China). All mice were handled in strict accordance with the Animal Ethics Procedures and Guidelines of the People's Republic of China, and the protocol of the present study was approved by the Institutional Committee for Animal Use at Sichuan University (SCU-2014-03). All mice were maintained under standard conditions, including a $12 \mathrm{~h}$ light/dark cycle at $18-22^{\circ} \mathrm{C}$ and $50-60 \%$ humidity, with ad libitum access to standard laboratory altromin chow (Dossy Co., Chengdu, China) and water.

The mice were randomly divided into three groups ( $\mathrm{n}=10$ per group) as follows: i) Normal control (N) group; ii) Porphyromonas gingivalis (PG) periodontal infection (P) group; and iii) hyperglycemia plus PG periodontal infection (HP) group. All mice were anesthetized using $220 \mathrm{mg} / \mathrm{kg}$ sodium pentobarbital (Anpro Pharmaceuticals, Arcadia, CA, USA) and were sacrificed via decapitation at 12-weeks-old.

Experimental diabetes induction. Diabetes was induced in 6-week-old HP group mice. Briefly, the mice in the HP group consumed a high-fat diet (48 kcal\% fat) (Dossy Co.) for 7 days, during which the mice received intraperitoneal injection with
$40 \mathrm{mg} / \mathrm{kg}$ STZ dissolved in buffer supplemented with citric acid ( $\mathrm{pH} 4.5$ ) for 5 days, followed by overnight fasting. After 7 days, the mice in the HP group received a standard low-fat diet (12.3 kcal\% fat; Dossy Co.) for the remaining experimental period. The mice in the $\mathrm{N}$ and $\mathrm{P}$ groups consumed a standard low-fat diet throughout the duration of the study. At 9 weeks old, all mice in the HP group were rendered diabetic, according to the standards described in a previous study (13).

Fasting blood glucose analysis. Blood glucose measurements were conducted when the mice were aged 9 weeks and upon sacrifice. Briefly, mouse tail veins were pierced using a needle, and the blood was collected from the tail vein following a $10-\mathrm{h}$ fast. A glucometer was used in order to determine the fasting blood glucose levels (OneTouch ${ }^{\circledR}$ Glucometer; LifeScan, Inc., Wayne, PA, USA).

Quantification of alveolar bone loss. Following sacrifice, the mandibular jaws of the mice were separated from the surrounding soft tissues. Alveolar bone loss at the first and second mandibular molars was monitored using a stereomicroscope with an attached digital camera (Leica MZ FLIII; Leica Microsystems GmbH, Wetzlar, Germany). The bone loss level was determined by measuring the area bordered by the cemento-enamel junction, alveolar bone crest, and mesial and distal line angles on the lingual side of the first and second mandibular molars. The measurements were conducted blindly using 15-fold magnified images of the bones and run in triplicate. Bone loss per animal was calculated as the average of both mandibles from each mouse.

Assessment of inflammatory cell infiltration. Monocyte and lymphocyte infiltration was determined as the number of cells in the alveolar bone biopsies, which were histologically prepared from all groups. Mouse maxillas were harvested following sacrifice and were prepared and examined using immunohistochemical staining, as previously described (14). Briefly, the maxillas were decalcified in $10 \%$ ethylene diaminetetraacetic acid (EDTA; Bio-Rad Laboratories, Inc.) for 14 days, and were subsequently embedded in paraffin. Samples were cut into $5 \mu \mathrm{m}$ sections and analyzed using immunohistochemical staining. Both sides of the maxillas were investigated and five slides were used for each sample at 10 intervals. Cell counts were conducted using images captured using a camera attached to a light microscope (Nikon Eclipse E600; Nikon Corporation, Tokyo, Japan) in a square of $45 \times 70 \mu \mathrm{m}$.

Isolation of murine meritoneal macrophages. Peritoneal macrophages were obtained by peritoneal lavage using $10 \mathrm{ml}$ ice-cold Hank's balanced salt solution supplemented with $10 \mathrm{U} / \mathrm{ml}$ heparin (both Gibco; Thermo Fisher Scientific, Inc., Waltham, MA, USA). The cells were washed twice, and resuspended in Dulbecco's modified Eagle's medium (DMEM) supplemented with $10 \%$ fetal bovine serum (FBS), penicillin and streptomycin (all Gibco; Thermo Fisher Scientific, Inc.). Subsequently the cells were seeded at a density of $2 \times 10^{6}$ cells/plate into $35-\mathrm{mm}$ plastic dishes (Sarstedt S.r.l, Verona, Italy). The plates were incubated in a humidified atmosphere containing $5 \% \mathrm{CO}_{2}$ at $37^{\circ} \mathrm{C}$ overnight to allow macrophage adherence. 
Isolation and culture of BMSCs. In order to isolate BMSCs, the femurs and tibias of the mice were harvested, and the muscles and extraosteal tissues were trimmed. BMSCs were centrifuged at $157 \mathrm{x} \mathrm{g}$ at $10^{\circ} \mathrm{C}$ for $5 \mathrm{~min}$ in a $1.073 \mathrm{~g} / \mathrm{ml}$ Percoll density gradient (GE Healthcare Bio-Sciences, Pittsburgh, PA, USA). After washing twice with phosphate-buffered saline (PBS), the cells were seeded into $25-\mathrm{cm}^{2}$ cell culture flasks containing L-DMEM, supplemented with $10 \%$ FBS and 1\% penicillin-streptomycin. Subsequently, the cultures were incubated at $37^{\circ} \mathrm{C}$ in $5 \% \mathrm{CO}_{2}$ for $48 \mathrm{~h}$. Following 7-10 days of culture, during which the original medium was replaced on day 4 , the cells were detached from the culture flask using $0.25 \%$ trypsin and $0.02 \%$ EDTA, and concentrated by centrifugation at $157 \mathrm{x} \mathrm{g}$ for $5 \mathrm{~min}$. Subsequently, the suspended BMSCs were seeded into 24 -well plates at a density of $1 \times 10^{6}$ cells/well and cultured for 2 days.

Transwell migration assay. BMSCs $\left(1 \times 10^{6}\right)$ were seeded into 24-well tissue culture plates containing DMEM. Macrophages $\left(3 \times 10^{5}\right)$ were seeded into a 24 -well Transwell ${ }^{\circledR}$ culture plate (8- $\mu \mathrm{m}$ pore size filter) containing $300 \mu \mathrm{l}$ media, and were exposed to BMSC conditioned media, which allowed the macrophages and BMSCs to grow in the same medium without direct contact. In addition, the BMSCs were exposed to macrophage conditioned media. All cells and the cell culture media were harvested following $48 \mathrm{~h}$ co-culture; the cells on the top of the porous membrane were manually scraped using a p200 pipette tip. The cells that had migrated to the underside were stained with crystal violet staining solution (Winchem Industrial Co., Ltd., Ningbo, China), which had been prepared as follows: Crystal violet was dissolved in methanol supplemented with $0.5 \%$ stock solution and further diluted with PBS in 1:4 dilutions for staining. Subsequently, images of the membrane were captured under the microscope (Leica Microsystems $\mathrm{GmbH}$ ) and the number of migrated cells were counted. Bound antibody was detected using the Supersignal West Pico Chemiluminescent Substrate system

Cytokine assays. Following 48-h co-culture, the culture supernatants were harvested, and the TNF- $\alpha$ level in the cell-free supernatants was quantified using the TNF- $\alpha$ commercial ELISA kit, according to the manufacturer's protocol. The concentration of TNF- $\alpha$ was determined by reference to a standard curve constructed with known amounts of mouse recombinant TNF- $\alpha$. The sensitivity limit for the assay was $8.0 \mathrm{pg} / \mathrm{ml} \mathrm{TNF}-\alpha$. The analysis was conducted using data from three independent experiments.

Immunohistochemical analysis. Maxillas were collected from the sacrificed mice and were used for immunohistochemical staining. Briefly, the maxillas were decalcified in 10\% EDTA solution for 14 days, after which they were embedded in paraffin. Thin sections $(5 \mu \mathrm{m})$ were cut and visualized using immunohistochemical staining, according to the manufacturer's protocol. Both sides of the maxillae were investigated and five slides were used for each sample at 10 intervals. For each sample, the average of the measurements from both sides were calculated. The primary antibodies included: Mouse anti-CCL2 (1:200), mouse anti-CCR2 (1:200) and anti-TNF- $\alpha$ (1:200). The secondary antibodies included anti-rabbit and anti-mouse horseradish peroxidase-conjugated antibodies $(1: 1,000)$. Images of the stained sections were captured using a microscope with an attached digital camera (Eclipse 80i; Nikon Corporation, Tokyo, Japan). The staining intensity was quantified using Image-Pro Plus 6.0 image analysis software (Media Cybernetics, Inc., Rockville, MD, USA), and was presented as the mean optical density.

Western blot analysis. The cells, including the macrophages on the porous membrane and the BMSCs in 24-well tissue culture plates, were washed with ice-cold PBS, after which total protein was extracted using the ReadyPrep Protein Extraction kit. Total protein $(\sim 30 \mu \mathrm{g})$ from each sample was separated using 5\% sodium dodecyl sulfate-polyacrylamide gel electrophoresis and transferred to nitrocellulose membranes by electroblotting. The membranes were incubated overnight at $4^{\circ} \mathrm{C}$ with primary antibodies against GAPDH (1:300), CCL2 (1:200), CCR2 (1:200), JNK (1:200), p-JNK (1:200), ERK (1:200), p-ERK (1:200), p38 (1:200) and p-p38 (1:200), followed by incubation with anti-rabbit $(1: 3,000)$ or anti-mouse $(1: 2,000)$ horseradish peroxidase-conjugated secondary antibodies for $1 \mathrm{~h}$ at room temperature. Antibody complexes were detected using the Super Signal West Pico Chemiluminescent Substrate.

Statistical analysis. Data are presented as the mean \pm standard deviation. SPSS statistical software, version 17.0 (SPSS, Inc., Chicago, IL, USA) was used for statistical analysis. Differences in parameter mean values between all groups were analyzed using one-way analysis of variance, followed by Student-Newman-Keuls test. $\mathrm{P}<0.05$ was considered to indicate a statistically significant difference.

\section{Results}

Alveolar bone loss and inflammatory cell infiltration. In order to determine the severity of periodontitis, the present study investigated alveolar bone loss and inflammatory cell infiltration in three groups by calculating the area $\left(\mathrm{mm}^{2}\right)$ bordered by the cemento-enamel junction, alveolar bone crest and mesial and distal line angles on the lingual sides of the first and second mandibular molars.

As presented in Fig. 1A, the mice in the HP and P groups exhibited markedly increased alveolar bone loss, as compared with the mice in the $\mathrm{N}$ group at sacrifice. In addition, the HP mice exhibited markedly increased alveolar bone loss, as compared with the P mice. Furthermore, the HP and P mice exhibited increased infiltration of inflammatory cells, as compared with the $\mathrm{N}$ mice (Fig. 1B).

Histomorphometrical analyses demonstrated that the expression levels of TNF- $\alpha$ in epithelia was significantly increased in the HP and Pg mice, as compared with the $\mathrm{N}$ mice; however, the HP mice exhibited significantly increased TNF- $\alpha$ expression levels, as compared with the $\mathrm{P}$ mice.

Cell migration analysis. Following $24 \mathrm{~h}$ incubation, the cells that had migrated to the lower side of the porous membrane were counted using the Transwell migration assay. As presented in Fig. 2, the HP mice exhibited significantly increased macrophage infiltration, as compared with $\mathrm{P}$ mice 

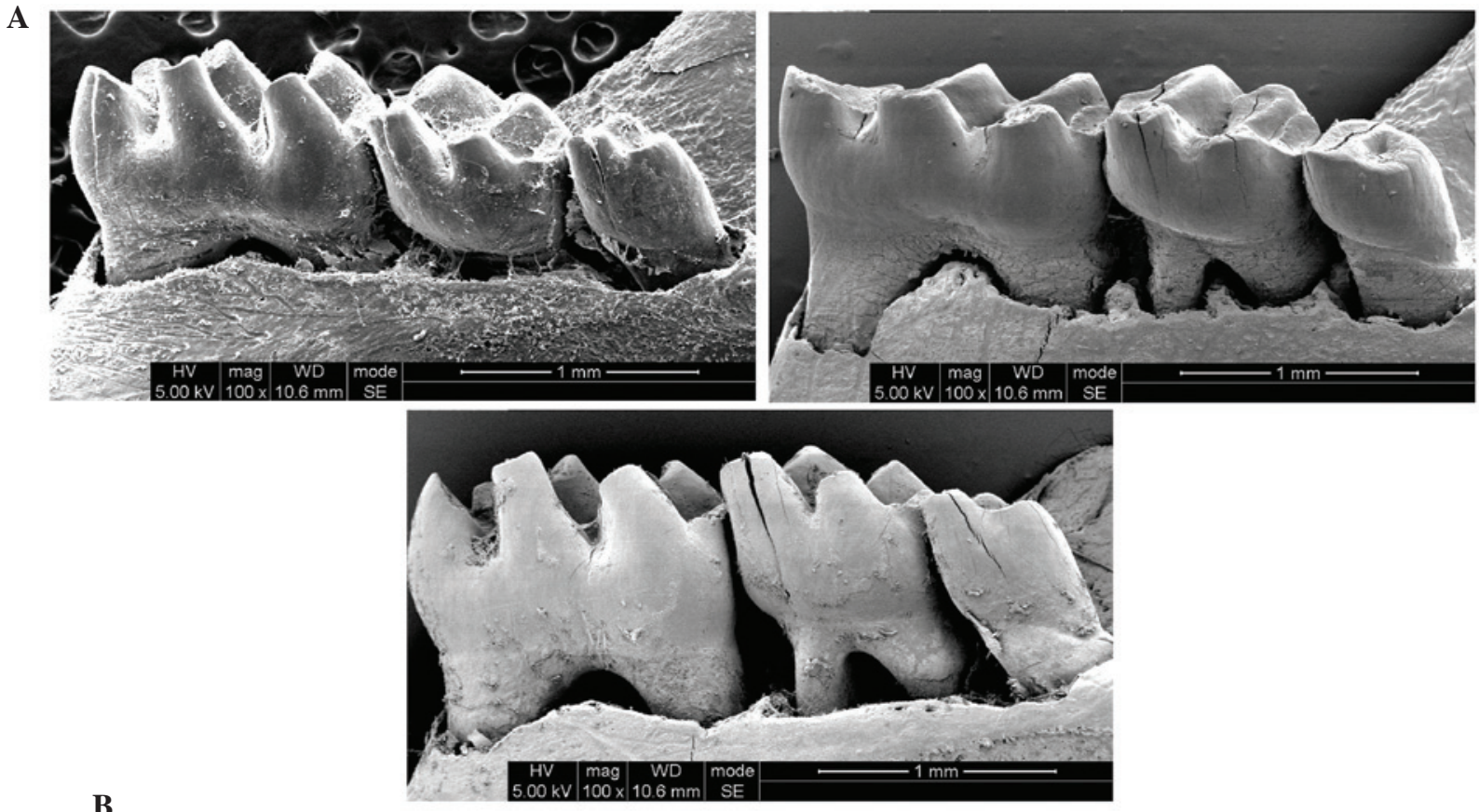

B
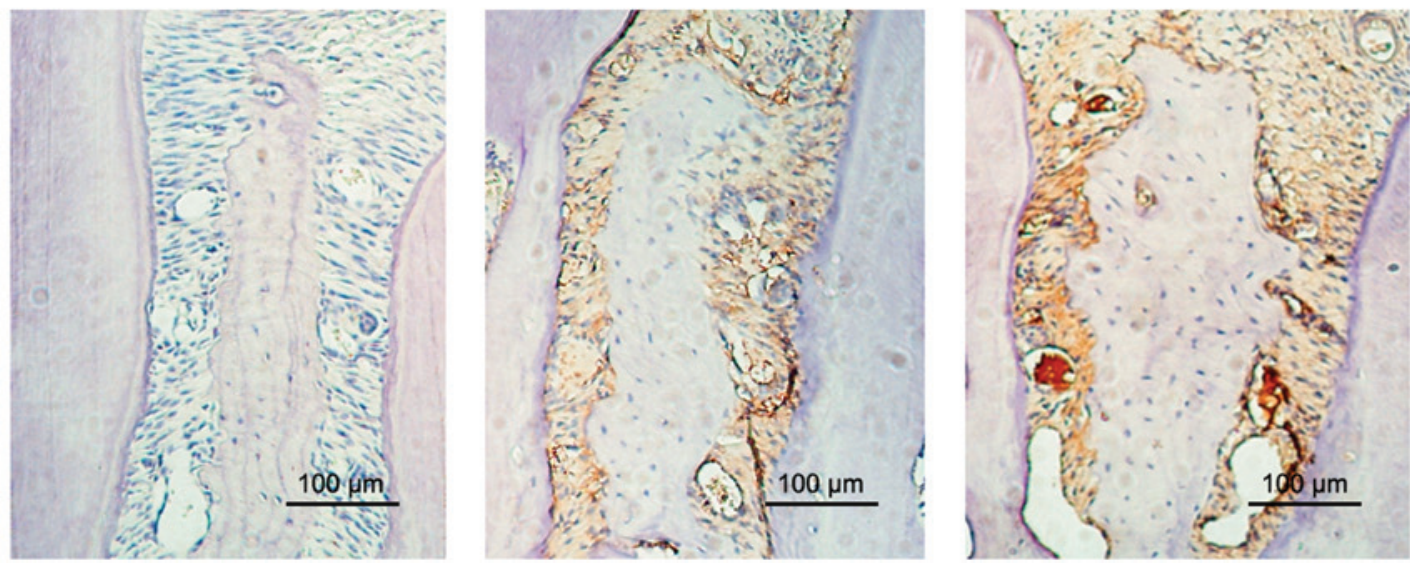

Figure 1. Alveolar bone loss and inflammatory cell infiltration. (A) Alveolar bone loss was measured using 15-fold magnified images of the mandibular bones. The data represent three independent experiments. (B) Immunohistochemical analysis of alveolar bone loss was conducted using a microscope with an attached digital camera (scale $=100 \mu \mathrm{m})$.

A

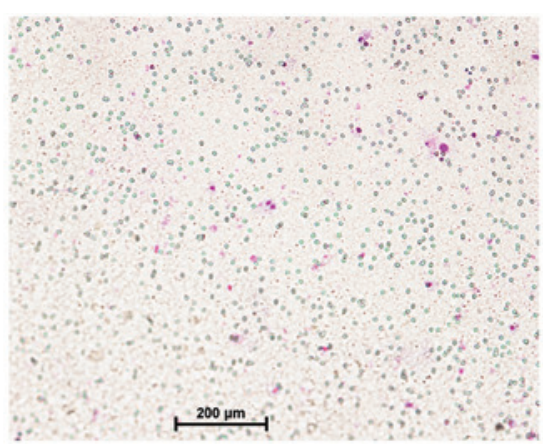

B

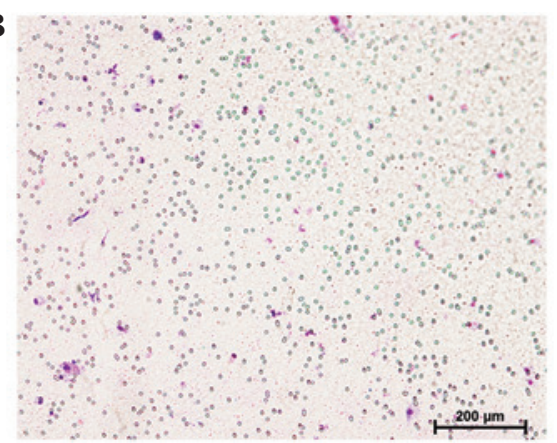

C

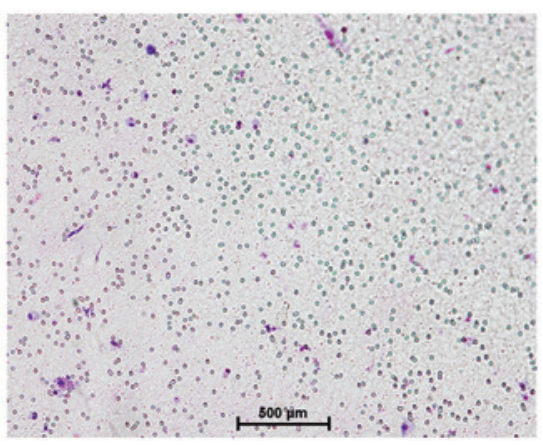

Figure 2. Isolation, co-culture and chemotaxis of murine meritoneal macrophages and bone marrow stem cells (BMSCs). The chemotaxis of macrophages and BMSCs in the (A) Porphyromonas gingivalis (PG) periodontal infection group (scale bar $=200 \mu \mathrm{m}$ ), (B) the hyperglycemia and PG periodontal infection group (scale bar=200 $\mu \mathrm{m}$ ), and (C) the normal control group (scale bar=500 $\mu \mathrm{m}$ ).

and the controls without treatment $(\mathrm{P}<0.05)$. No significant differences were detected between $\mathrm{P}$ mice and the normal controls $(\mathrm{P}>0.05)$.
Levels of TNF- $\alpha$ in vivo. The levels of TNF- $\alpha$ in the cell-free supernatants were determined using an ELISA. As presented in Table I, the levels of TNF- $\alpha$ in the HP and Pg groups 
Table I. TNF- $\alpha$ levels in the cell supernatant.

\begin{tabular}{lc}
\hline Group $(\mathrm{n}=3)$ & TNF- $\alpha(\mathrm{pg} / \mathrm{ml})$ \\
\hline HP & $167.3 \pm 10.27^{\mathrm{a}}$ \\
P & $68.7 \pm 5.9^{\mathrm{b}}$ \\
Normal control & $20.4 \pm 3.1^{\mathrm{c}}$
\end{tabular}

${ }^{\mathrm{a}} \mathrm{P}>0.05$, as compared with the $\mathrm{P}$ mice group; ${ }^{\mathrm{b}} \mathrm{P}>0.05$, as compared with the normal control group; ${ }^{\mathrm{C}} \mathrm{P}>0.05$, as compared with the HP mice group. HP, hyperglycemia plus Porphyromonas gingivalis infection group; P, Porphyromonas gingivalis periodontal infection group. TNF- $\alpha$, tumor necrosis factor- $\alpha$.

CCL2

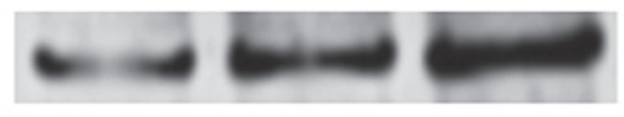

CCR2

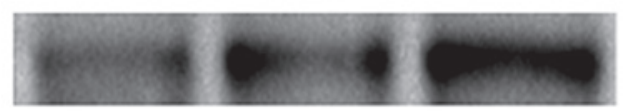

JNK

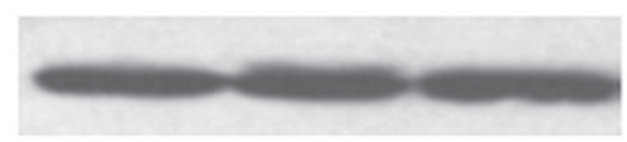

pERK

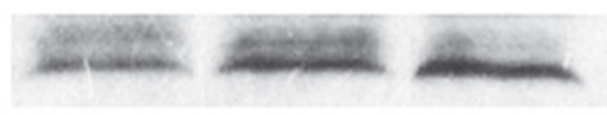

pp38

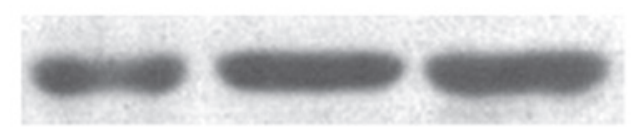

GAPDH

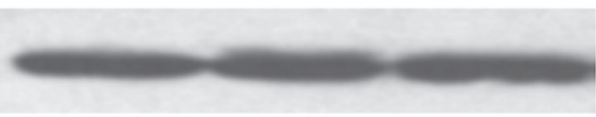

Figure 3. Protein expression levels of CCL2, CCR2, JNK, and p-ERK and p-p38 were examined in the three groups of mice using western blotting. The bands were quantified, and the ratios between the expression levels of the target protein and GAPDH were calculated for statistical analysis. CCL2, chemokine (C-C Motif) ligand 2; CCR2, chemokine (C-C Motif) receptor 2; JNK, c-Jun N-terminal kinase; p-ERK, phosphorylated-extracellular signal-regulated kinase; GAPDH, glyceraldehyde-3-phosphate dehydrogenase.

were significantly increased, as compared with the $\mathrm{N}$ group $(\mathrm{P}<0.05)$; however, the TNF- $\alpha$ level was significantly higher in the HP group, as compared with the Pg group.

Protein expression levels. In order to investigate the underlying mechanism of alveolar bone loss in DP, the protein expression levels of CCL2, ERK, p-ERK, JAK, p-JAK, p38 and p-p38 in the BMSCs, and CCR2 in the macrophages, were evaluated by western blot. As presented in Fig. 3, the protein expression levels of CCL2, p-ERK, p-JAK and p-p38 in the BMSCs were significantly increased in the HP and Pg groups, as compared with the $\mathrm{N}$ group $(\mathrm{P}<0.05)$; however, this increase was most significant for the HP group. Conversely, there was no significant difference in the protein expression levels of ERK, JAK, p38 between the three groups $(\mathrm{P}>0.05)$. In addition, the protein expression levels of CCR2 in the macrophages were significantly increased in the HP and Pg groups, as compared with the $\mathrm{N}$ group $(\mathrm{P}<0.05)$, and this increase in CCR2 expression levels was most significant in the HP group.

\section{Discussion}

DP is typically associated with severe clinical symptoms that make its treatment more challenging, as compared with PD in the absence of diabetes (13-15). Disorders of the inflammatory response are thought to be crucially involved in the progression of DP (16). Furthermore, significant alveolar bone loss was detected in mice with untreated DP associated with high TNF- $\alpha$ levels which can negatively regulate insulin tolerance, pancreatic islets and periodontal tissues (17-19). Previous studies demonstrated that infection or hyperglycemia contributed to the increased production of TNF- $\alpha$ by monocytes and macrophages, and that excessive TNF- $\alpha$ generation in turn promoted a higher proinflammatory state and tissue damage $(20,21)$. In the present study, the mice with untreated DP exhibited the most significantly increased levels of TNF- $\alpha$; thus suggesting that periodontal pathogens and hyperglycemia exerted a synergistic effect on TNF- $\alpha$ production. This is consistent with previous results of studies involving diabetic patients and patients with PD, who were shown to exhibit high serum levels of TNF- $\alpha(22,23)$.

CCL2 is a C-C group chemokine that is expressed by numerous cells, including leukocytes, fibroblasts, kerotinocytes and endothelial cells, in response to various endogenous and exogenous stimuli $(24,25)$. The present study demonstrated that high levels of glucose and inflammation increased the activation of MAPK signaling and upregulated the expression levels of CCL2 in BMSCs (a representative chemokine for the migration of macrophages via the receptor CCR2) (26). These results, in combination with the increased infiltration of macrophages into the periodontal of DP mice and histological analyses, suggested that macrophages were the predominant source of TNF- $\alpha$ in response to PG infection and high glucose levels. In addition, these results suggested that the inflamed periodontium was infiltrated by macrophages.

The migration of monocytes and macrophages to sites of inflammation may promote inflammatory tissue destruction due to the requirement of dynamic integrin-dependent adhesion. The present study demonstrated that PG infection and high glucose levels stimulated BMSC-mediated synthesis of CCL2, which may have contributed to the migration of macrophages into the inflamed tissue via the CCL2/CCR2 axis; thus suggesting that the increased infiltration of macrophages and higher levels of TNF- $\alpha$ in the DP mice may have led to the severe alveolar bone loss.

In conclusion, the results of the present study suggested that DP may be characterized as an interaction between local inflammation and the host immune responses, in particular involving the upregulation of CCL 2 and TNF- $\alpha$ expression levels in periodontal tissues. In addition, it may be characterized by the recruitment of macrophages via the CCL2/CCR2 axis, which in turn may lead to the alveolar bone loss associated with DP. 


\section{References}

1. Papadopoulos G, Weinberg EO, Massari P, Gibson FC III, Wetzler LM, Morgan EF and Genco CA: Macrophage-specific TLR2 signaling mediates pathogen-induced TNF-dependent inflammatory oral bone loss. J Immunol 190: 1148-1157, 2013.

2. Veronica L, Saviuc CM and Chifiriuc MC: Periodontitis and Periodontal Disease - innovative strategies for reversing the chronic infectious and inflammatory condition by natural products. Curr Pharm Des: Nov 12, 2015 (Epub ahead of print).

3. Graves D: Cytokines that promote periodontal tissue destruction. J Periodontol 79 (Suppl): 1585-1591, 2008.

4. Herrera BS, Bastos AS, Coimbra LS, Teixeira SA, Rossa C Jr, Van Dyke TE, Muscara MN and Spolidorio LC: Peripheral blood mononuclear phagocytes from patients with chronic periodontitis are primed for osteoclast formation. J Periodontol 85: e72-81, 2014

5. Hernández M, Gamonal J, Salo T, Tervahartiala T, Hukkanen M, Tjäderhane L and Sorsa T: Reduced expression of lipopolysaccharide-induced CXC chemokine in Porphyromonas gingivalis-i nduced experimental periodontitis in matrix metalloproteinase- 8 null mice. J Periodontal Res 46: 58-66, 2011.

6. Sakallioğlu EE, Ayas B, Lütfioğlu M, Keleş GC, Açikgöz G and Firatli E: Gingival levels of monocyte chemoattractant protein-1 (MCP-1) in diabetes mellitus and periodontitis: An experimental study in rats. Clin Oral Investig 12: 83-89, 2008.

7. Rauner M, Stein N, Winzer M, Goettsch C, Zwerina J, Schett G, Distler JH, Albers J, Schulze J, Schinke T, et al: WNT5A is induced by inflammatory mediators in bone marrow stromal cells and regulates cytokine and chemokine production. J Bone Miner Res 27: 575-585, 2012.

8. Shin Y, Han S, Jeon JS, Yamamoto K, Zervantonakis IK, Sudo R, Kamm RD and Chung S: Microfluidic assay for simultaneous culture of multiple cell types on surfaces or within hydrogels. Nat Protoc 7: 1247-1259, 2012.

9. Wu J, Chen Q, Liu W, Zhang Y and Lin JM: Cytotoxicity of quantum dots assay on a microfluidic 3D-culture device based on modeling diffusion process between blood vessels and tissues. Lab Chip 12: 3474-3480, 2012.

10. Gao D, Liu H, Lin JM, Wang Y and Jiang Y: Characterization of drug permeability in Caco-2 monolayers by mass spectrometry on a membrane-based microfluidic device. Lab Chip 13: 978-985, 2013.

11. Huh D, Matthews BD, Mammoto A, Montoya-Zavala M, Hsin HY and Ingber DE: Reconstituting organ-level lung functions on a chip. Science 328: 1662-1668, 2010.

12. Achyuta AK, Conway AJ, Crouse RB, Bannister EC, Lee RN, Katnik CP, Behensky AA, Cuevas J and Sundaram SS: A modular approach to create a neurovascular unit-on-a-chip. Lab Chip 13: 542-553, 2013.
13. Graves DT and Kayal RA: Diabetic complications and dysregulated innate immunity. Front Biosci 13: 1227-1239, 2008.

14. Sulniute R, Lindh T, Wilczynska M, Li J and Ny T: Plasmin is essential in preventing periodontitis in mice. Am J Patho 179: 819-828, 2011.

15. Lamster IB, Lalla E, Borgnakke WS and Taylor GW: The relationship between oral health and diabetes mellitus. J Am Dent Assoc 139 (Suppl): 19S-24S, 2008.

16. Preshaw PM: Periodontal disease and diabetes. J Dent 37: S575-S577, 2009.

17. Ekuni D, Tomofuji T, Irie K, Kasuyama K, Umakoshi M, Azuma T, Tamaki N, Sanbe T, Endo Y, Yamamoto T, et al: Effects of periodontitis on aortic insulin resistance in an obese rat model. Lab Invest 90: 348-359, 2010.

18. Gurzov EN and Eizirik DL: Bcl-2 proteins in diabetes: Mitochondrial pathways of $\beta$-cell death and dysfunction. Trends Cell Biol 21: 424-431, 2011.

19. Graves DT, Oskoui M, Volejnikova S, Naguib G, Cai S, Desta T, Kakouras A and Jiang Y: Tumor necrosis factor modulates fibroblast apoptosis, PMN recruitment, and osteoclast formation in response to $P$. gingivalis infection. J Dent Res 80: 1875-1879, 2001.

20. Gonzalez Y, Herrera MT, Soldevila G, Garcia-Garcia L, Fabián G, Pérez-Armendariz EM, Bobadilla K, Guzmán-Beltrán S, Sada E and Torres M: High glucose concentrations induce TNF- $\alpha$ production through the down-regulation of CD33 in primary human monocytes. BMC Immunol 13: 19, 2012.

21. Amar S, Oyaisu K, Li L and Van Dyke T: Moesin: A potential LPS receptor on human monocytes. J Endotoxin Res 7: 281-286, 2001.

22. Lechleitner M, Koch T, Herold M, Dzien A and Hoppichler F: Tumour necrosis factor-alpha plasma level in patients with type 1 diabetes mellitus and its association with glycaemic control and cardiovascular risk factors. J Intern Med 248: 67-76, 2000.

23. Górska R, Gregorek H, Kowalski J, Laskus-Perendyk A, Syczewska $\mathrm{M}$ and Madaliński K: Relationship between clinical parameters and cytokine profiles in inflamed gingival tissue and serum samples from patients with chronic periodontitis. J Clin Periodontol 30: 1046-1052, 2003.

24. Sassy-Prigent C, Heudes D, Mandet C, Bélair MF, Michel O, Perdereau B, Bariéty J and Bruneval P: Early glomerular macrophage recruitment in streptozotocin-induced diabetic rats. Diabetes 49: 466-475, 2000.

25. Nomura S, Shouzu A, Omoto S, Nishikawa M and Fukuhara S: Significance of chemokines and activated platelets in patients with diabetes. Clin Exp Immunol 121: 437-443, 2000.

26. Saeki K, Kanai T, Nakano M, Nakamura Y, Miyata N, Sujino T, Yamagishi Y, Ebinuma H, Takaishi H, Ono Y, et al: CCL2-induced migration and SOCS3-mediated activation of macrophages are involved in cerulein-induced pancreatitis in mice. Gastroenterology 142: 1010-1020.e9, 2012. 